1 Mestranda em Saúde Pública pela Universidade Estadual do Ceará (Uece) Fortaleza (CE), Brasil.

julianapessoato@hotmail.com

2 Doutora em Enfermagem pela Universidade de São Paulo (USP) - São Paulo (SP), Brasil. Professora Titular da Universidade Estadual do Ceará (Uece) Fortaleza (CE), Brasil. maria.salete.jorge@gmail.com

${ }^{3}$ Doutoranda em Saúde Coletiva pela Universidade Estadual do Ceará (Uece) - Fortaleza (CE), Brasil e Universidade Federal do Ceará (UFC) - Fortaleza (CE), Brasil.

mardeniagomes@yahoo.com.br

4 Doutoranda em Saúde Coletiva pela Universidade Estadual do Ceará (Uece)

- Fortaleza (CE), Brasil e Universidade Federal do Ceará (UFC) - Fortaleza (CE), Brasil.

psicoim@hotmail.com

5 Doutoranda em Saúde Coletiva pela Universidade Estadual do Ceará (Uece)

- Fortaleza (CE), Brasil e Universidade Federal do Ceará (UFC) - Fortaleza (CE), Brasil.

indaracavalcante@yahoo.com.br

\section{Resolubilidade do cuidado na atenção primária: articulação multiprofissional e rede de serviços}

\author{
Solvability of the caretaking in primary care: multiprofessional \\ articulation and services network
}

Juliana Pessoa Costa', Maria Salete Bessa Jorge ${ }^{\mathbf{2}}$, Mardenia Gomes Ferreira Vasconcelos ${ }^{\mathbf{3}}$, Milena Lima de Paula $\mathbf{4}$, Indara Cavalcante Bezerra $\mathbf{5}$

RESUMO Objetivou-se discutir a resolubilidade do cuidado em saúde na atenção primária a partir dos discursos de profissionais que atuam nas equipes da Estratégia Saúde da Família (ESF) e nos Núcleos de Apoio à Saúde da Família (Nasf), nos municípios de Maracanaú e Fortaleza, ambos no estado do Ceará. Os resultados indicam que os profissionais relacionam resolubilidade à ação realizada por uma equipe multiprofissional, com produção de vínculo e autonomia no processo de trabalho. No entanto, a rotatividade dos profissionais, a centralidade no trabalho médico e a insuficiente utilização da contrarreferência no fluxo de serviços são entraves no cotidiano da atenção primária.

PALAVRAS-CHAVE Atenção Primária à Saúde; Resolução de problemas; Assistência à saúde.

ABSTRACT It was aimed to discuss the solvability of the caretaking in health in the primary care from the speech of professionals who work in the. Family Health Strategy (ESF) teams and in the Nuclei of Support for Family Health (Nasf), in the municipalities of Maracanaú and Fortaleza, both in the state of Ceará. The results indicate that the professionals have associated solvability to the action carried out by a multiprofessional team, with production of bonds and autonomy in the work process. However, professionals turnover, the centrality in medical work and the deficient use of the counter reference in the flow of services are obstacles in the day-to-day of primary care.

KEYWORDS Primary Health Care; Problem solving; Health assistance. 


\section{Introdução}

No Brasil, o Sistema Único de Saúde (SUS), consolidado a partir da Constituição Federal de 1988, ao agregar os serviços da União, dos estados, dos municípios e da assistência médica previdenciária do antigo Instituto Nacional de Assistência Médica da Previdência Social (Inamps), promoveu a formação de redes assistenciais descentralizadas, regionalizadas e integradas, com o objetivo de garantir o acesso à saúde (SILVA, 2011).

Nesse contexto, em que se busca a universalização da saúde, as redes de assistência podem ser consideradas organizações poliárquicas de conjuntos de serviços de saúde, vinculados entre si por uma missão única, com objetivos comuns e ações cooperativas e interdependentes, que permitem ofertar uma atenção contínua (MENDES, 2010).

No entanto, a garantia do acesso a serviços assistenciais não implica a efetivação do cuidado em saúde. Nesse sentido, a integralidade preconiza que, além de atividades de promoção, prevenção e tratamentos, a forma como as práticas de cuidado são realizadas nos serviços de saúde também é fundamental para o alcance da resolubilidade do atendimento. Portanto, as ações devem ser realizadas de forma humanizada, ou seja, os profissionais devem perceber o usuário como um sujeito com subjetividades (LIMA ET AL., 2012).

Dessa forma, para assegurar a qualidade da assistência nos três níveis de atenção à saúde, devem-se considerar as relações interpessoais no processo de cuidado. $\mathrm{O}$ acolhimento deve ser uma prática valorizada pelos profissionais, pois possibilita atender às reais demandas dos usuários. Quanto à organização da equipe, preza-se pelo formato multiprofissional, ao possibilitar uma visão mais ampla do sujeito e a superação do modelo biomédico (RODRIGUES ET AL., 2013).

Nesse prisma, o trabalho em equipe multiprofissional possibilita a construção de um trabalho cooperativo a partir de múltiplas intervenções técnicas e interação de sujeitos com diferentes profissões, permitindo um 'fazer' de forma integrada, por meio da articulação das ações multiprofissionais e da cooperação (FERREIRA ET AL. 2009).

Além de uma equipe multiprofissional, também é necessário valorar as relações interpessoais dos integrantes dessa equipe. Desse modo, é imprescindível que haja a formação de vínculos profissionais, o que possibilita aos trabalhadores compreender a realidade a ser vivenciada no sentido de promover o crescimento de cada um e de todo o grupo de trabalho. Esse processo facilita a resolução de conflitos existentes na prática cotidiana. Porém, para que haja essa formação de vínculos, o grupo de trabalho deve ser maleável, receptivo e adaptável às contínuas modificações que ocorrem no serviço (MARTINS ET AL., 2012).

Diante do exposto, percebe-se que questões relacionadas à forma como a rede assistencial está organizada e como se constitui a qualidade do atendimento ofertado ao usuário estão associadas a um cuidado integral e também à resolutividade do serviço (QUINDERÉ, 2013). O conceito de resolutividade está associado à resolução final dos problemas trazidos pelos usuários ao serviço e à satisfação desses e dos profissionais (TURRINI ET AL., 2008).

Considera-se resolutividade a resposta satisfatória que o serviço fornece ao usuário quando busca atendimento a alguma necessidade de saúde. Essa resposta não compreende, exclusivamente, a cura de doenças, mas, também, o alívio ou a minimização do sofrimento, a promoção e a manutenção da saúde. Por sua vez, a resolutividade pode ser avaliada em dois aspectos: no próprio serviço, quanto à capacidade de atender à sua demanda e no encaminhamento dos casos que necessitam de atendimentos mais especializados dentro do sistema de saúde, que se estendem desde a consulta inicial, os exames e o tratamento do usuário no serviço de Atenção Primária à Saúde (APS) até a 
solução de seu problema em outros níveis de atenção (TURRINI ET AL., 2008).

Em face dessas questões referentes à resolubilidade dos serviços, o objeto deste estudo mostra-se relevante para contribuir para a formulação de práticas mais condizentes com as demandas dos usuários referentes a trabalhos em saúde, acesso e satisfação, resolutividade e qualidade do serviço. Nesse contexto, objetivou-se avaliar a resolubilidade do cuidado em saúde na atenção primária, a partir dos discursos de profissionais do Nasf e da ESF.

\section{Metodologia}

Optou-se pela abordagem qualitativa, que busca entender o universo de significados, valores e crenças, os quais não podem ser quantificados e onde as relações entre os indivíduos não podem ser reduzidas à operacionalização de variáveis (MINAYO, 2008). O estudo integra uma pesquisa mais ampla, denominada Produção do cuidado na Estratégia Saúde da Família e sua interface com a saúde mental: os desafios da resolubilidade, financiada pelo Conselho Nacional de Desenvolvimento Científico e Tecnológico (CNPq).

Utilizaram-se quatro unidades da ESF como cenário, localizadas no município de Fortaleza, Ceará, onde atuam equipes de saúde da família e Nasf. Essa cidade foi intencionalmente selecionada para o estudo em virtude de estar pactuada pelo Sistema Municipal de Saúde Escola e de integrar a referida pesquisa, como instituição coparticipante. Esses cenários fazem parte da área de corresponsabilidade sanitária com a saúde da população da instituição de ensino responsável pela investigação.

Os participantes da pesquisa foram constituídos por 20 profissionais de saúde que compunham equipes de ESF e Nasf. Para a análise, foram utilizados os discursos de enfermeiros, dentistas, médicos, fisioterapeutas e fonoaudiólogos. Como critérios de inclusão dos participantes, definiu-se: estar vinculado a uma equipe multiprofissional dos serviços na atenção primária, seja ao Nasf ou à ESF, com, no mínimo, um ano de atuação.

Esses profissionais foram abordados na unidade de saúde à qual estavam vinculados, respondendo a entrevista semiestruturada, com temas relacionados à resolubilidade do cuidado e à articulação do trabalho na atenção primária com os demais níveis de complexidade do SUS. As entrevistas foram gravadas com permissão dos participantes e tiveram duração média de 30 minutos.

Antes da realização do trabalho de campo, o estudo foi submetido à análise do Comitê de Ética em Pesquisa com Seres Humanos (CEP) da Universidade Estadual do Ceará (Uece), recebendo indicação favorável com parecer $n^{\circ}$ 10245206-7. Os sujeitos tiveram acesso ao Termo de Consentimento Livre e Esclarecido, o qual assinaram, autorizando sua participação na pesquisa, conforme as recomendações do Conselho Nacional de Saúde.

Para organização das informações, seguiram-se três etapas estabelecidas por Minayo (1999) e retraduzidas por Assis e Jorge (2010): ordenação, classificação e análise final dos dados, que incluem classificação das falas dos entrevistados, componentes das categorias empíricas, sínteses horizontal e vertical, e confronto entre as informações, agrupando as ideias convergentes, divergentes e complementares.

A leitura exaustiva do material proporcionou o estabelecimento das categorias emergentes das falas com relação aos assuntos tratados na entrevista. A análise final foi orientada pela análise de conteúdo, com base em Minayo (2008). A autora enfatiza que, entre as possibilidades de categorização no campo da saúde, a mais utilizada é a análise de conteúdo temática, consistindo em isolar temas de um texto e extrair as partes utilizáveis, de acordo com o tema pesquisado, para permitir comparação com outros textos escolhidos da mesma maneira. 


\section{Resultados e discussão}

Os resultados apresentados na presente pesquisa foram discutidos em duas temáticas: (1) O trabalho na equipe multiprofissional: autonomia para resolubilidade da assistência; (2) Encaminhamentos e dificuldades na contrarreferência: (des)articulação dos níveis de complexidade do SUS.

\section{O trabalho na equipe multiprofissio- nal: autonomia para resolubilidade da assistência}

A investigação possibilitou apreender que a resolubilidade das ações de saúde está implicada no trabalho efetivo da equipe multiprofissional, que busca solucionar os problemas individuais e coletivos demandados pela população. No entanto, os profissionais enfrentam dificuldades quanto à formação de uma equipe completa, às condições de trabalho $\mathrm{e}$ à regência do modelo biomédico, ainda imperativo na rede de serviços ofertada.

O discurso do profissional da equipe de saúde da família, a seguir, evidencia a importância do trabalho compartilhado:

O trabalho em equipe multiprofissional é mais resolutivo. [...] o enfermeiro, sozinho, não vai resolver o problema da comunidade. [...] Atender em parceria com o Nasf, com o médico, com toda uma equipe multidisciplinar que vai atender pra conseguir uma melhor assistência da qualidade prestada. (Enfermeira, ESF).

No campo empírico, está evidente que o trabalho em equipe, para o profissional, é uma prática colaborativa de troca de saberes e experiências, que possibilita maior resolutividade para os problemas apresentados pela comunidade. Essa concepção de equipe otimiza as habilidades entre seus membros, compartilha a gestão de casos e proporciona melhores serviços de saúde aos pacientes e à comunidade (NUNES ET AL., 2012).

De modo complementar, os profissionais referem que a equipe multiprofissional conecta diferentes saberes, estabelece vínculos com base no conhecimento do trabalho do outro. Isso promove o entrosamento entre os profissionais e a valorização da sua participação na produção de cuidados, tornando o trabalho mais resolutivo:

O vínculo entre as equipes multiprofissionais e dos serviços o torna mais resolutivo. [...] Os fisioterapeutas, assistentes sociais, psicólogos sempre tentam se unir, também, aos médicos e enfermeiros para realizar atividades em grupo [...] então, a gente tem diferentes saberes reunidos. (Dentista, ESF).

Com efeito, para que o cuidado em saúde seja resolutivo, a equipe multiprofissional deve estar completa para atuar na ESF e no Nasf. No território, observou-se que a equipe de saúde da família, em especial, quando incompleta, dificulta o serviço e o cuidado em saúde, como observado na fala dos profissionais:

[...] temos algumas dificuldades por não ter um quadro completo de profissionais [...] em virtude da demanda e do reduzido número de profissionais nas unidades mais complexas, faz com que haja uma grande fila de espera. (Enfermeira ESF).

[...] o SUS é uma política muito bonita, mas ainda precisa de algumas soluções. Falta médico em duas equipes. Enfermeiro está completo, mas faltam também insumos. (Enfermeiro ESF).

Uma equipe multiprofissional incompleta revela a crise da situação de trabalho dos profissionais de saúde que atuam no SUS, ocasionando aumento de filas e demora no atendimento aos usuários.

Nesse contexto, observa-se que os discursos dos participantes convergem na ideia, de que a falta de um profissional em uma equipe dificulta o cuidado integral na 
atenção primária, comprometendo a resolubilidade da assistência prestada. Outro aspecto percebido, a partir das narrativas dos profissionais, tanto da equipe do Nasf, como da ESF, foi a ênfase no trabalho do médico em detrimento da articulação em equipe. Nesse contexto, os outros profissionais ficam com suas ações restritas, em virtude da prescrição e dos encaminhamentos do médico. Isso representa a inversão da proposta da ESF de mudança do modelo de cuidado, reafirmando a prática da clínica biologicista e centralizada no fazer médico.

[...] Nós ficamos um pouco restritos. Quem pode prescrever e dar a indicação é o médico, quando a gente precisa de um exame na atenção secundária [...] então, a gente tem que estar falando com o médico pra ele prescrever o exame ou outro tratamento. (Fisioterapeuta Nasf).

[...] a carga horária do médico não está sendo cumprida. A partir do momento em que o médico não está na mesma carga horária que eu, dificulta a continuidade do meu atendimento. (Enfermeira ESF).

Deste modo, o trabalho em saúde vai se constituindo a partir de relações de poder reveladas pelo saber médico enquanto corpo de conhecimento estruturado e da sua representação social diante da sociedade. Assim, o trabalho não é desenvolvido em conjunto, mas, sim, isoladamente, fato que prejudica uma prática que deveria ser alicerçada na interdisciplinaridade. No cotidiano, reproduz-se uma atuação compartimentada com forte tendência a reproduzir o modelo médico-centrado (MEDEIROS ET AL., 2010).

Questiona-se, assim, a autonomia no processo de trabalho dos profissionais que fazem parte das equipes de ESF e Nasf. Como esses profissionais podem transpor o modelo de cuidado em saúde, se estão operando numa lógica de fragmentação e divisão técnica do trabalho na atenção primária?
Como empoderar e conduzir a equipe à participação em projetos de cuidado ampliados e articulados com os níveis de complexidade do SUS?

Para contextualizar tal problemática, é importante entender como está disposta a rede de serviços e sua articulação na atenção primária. A ESF baseia-se na organização multiprofissional para a produção da saúde, a partir de uma abordagem que estimula à ação os diversos saberes e práticas dos profissionais. Portanto, o trabalho em equipe significa a interação entre os diversos profissionais, (um dos pilares deste modelo de atenção) e, a busca da integralidade nos cuidados de saúde e na relação entre a complementaridade de trabalhos (PIRES; CAMPOS, 2010).

O trabalho em equipes multiprofissionais na ESF tornou-se fundamental no cuidado em saúde, pois, com o processo de mudança do modelo assistencial no contexto do SUS, ocorreu a ampliação do objeto de trabalho, ou seja, da doença para o indivíduo e sua família. Essa ampliação no objeto de intervenção, para além dos âmbitos individual e clínico, exigiu mudanças na forma de atuação e na organização do trabalho, bem como demanda alta complexidade de ações mediante a interação de diversos saberes e práticas (COSTA ET AL., 2012).

O trabalho em equipe, nesse contexto, é considerado importante, porém difícil em sua gestão. Deve ser visto como uma maneira de dividir as responsabilidades e alcançar intervenções efetivas e singulares no processo saúde-doença do usuário. A eficiência e a eficácia dos serviços, nessa perspectiva, requerem modalidade de trabalho em equipe que traduza a forma de conectar as diferentes ações e os distintos profissionais de saúde (SCHRAIBER ET AL., 2010).

Desse modo, a noção de equipe multiprofissional é tomada como uma realidade exigida, uma vez que existem profissionais de diferentes áreas atuando conjuntamente com um único objetivo, que é a integralidade da atenção em saúde. A proposta do trabalho 
em equipe, nesse contexto, consistirá numa modalidade de trabalho coletivo baseada em relações recíprocas entre as intervenções técnicas e as diversas áreas de conhecimento de cada núcleo profissional (LIMA ET AL., 2012).

Num sentido mais amplo, a organização multiprofissional na atenção primária inclui as equipes dos núcleos de atenção à saúde da família. Estes, por sua vez, foram criados e implantados com o intuito de apoiar a melhor articulação da ESF na rede de serviços em saúde. Além disso, os Nasfs são responsáveis por realizar ações conjuntas às equipes da ESF, intervenções coletivas de promoção, prevenção e acompanhamento de grupos sociais em vulnerabilidade no contexto do território (ANDRADE ET AL., 2012).

Assim, a ESF e o Nasf constituem a rede básica de atenção à saúde, que deve funcionar como uma das portas de entrada do conjunto de serviços que compõem o SUS. Nesse nível de atenção, ações simples e de baixa incorporação tecnológica corresponderiam a graus elevados de resolubilidade da maioria dos problemas de saúde, que, com retaguardas de maior complexidade, complementariam a resolução dos problemas mais compleXOS (MERHY; ONOCKO, 1997).

Dessa forma, os profissionais das equipes da ESF e do Nasf são corresponsáveis por uma população adscrita, que tem como objetivo o enfrentamento dos diferentes fatores que interferem no processo saúde-doença. A ação da equipe multiprofissional deverá possibilitar que um profissional se reconstrua a partir da prática do outro, ambos sendo transformados para uma intervenção na realidade em que estão inseridos (MARTINS ET AL., 2012).

Para tamanha mobilidade e dinamismo, é patente que a interação (integração e compartilhamento de saberes) entre os profissionais, e entre estes e os usuários, aconteça durante todo o processo de cuidado. Na prática, entretanto, a realidade encontrada depara-se com uma equipe de especialidades variadas, porém, compartimentadas, com pouca comunicação, dificultando a resolubilidade dos problemas.

Pode-se relacionar a dificuldade em manter completa a equipe de saúde da família ou do Nasf com as insuficientes condições de trabalho dos profissionais do serviço público, além da falta de recursos técnicos, da precariedade dos equipamentos existentes, da inadequação do espaço físico e dos materiais (SHIMIZU; CARVALHO JUNIOR, 2012). Além desses fatores, os baixos salários, a ausência de apoio dos gestores, o insuficiente reconhecimento social, o débil desenvolvimento da carreira, as diversas formas de contratos, sobretudo os informais, contribuem para a fragilidade de vínculos com o serviço e a alta rotatividade de profissionais ( $\mathrm{POZ}, 2013)$.

A essa condição, soma-se a realidade de que os profissionais de saúde enfrentam precários ambientes de trabalho. Nesse sentido, Pinto et al. (2010) afirmam que a remuneração insuficiente para o atendimento das suas necessidades dificulta a manutenção do vínculo trabalhista. Os profissionais da saúde enfrentam o desafio de ter ou não seu contrato de trabalho anual renovado. A instabilidade do vínculo trabalhista e a insegurança são os principais responsáveis pela alta rotatividade dos profissionais que atuam nas equipes da ESF (PINTO ET AL., 2010).

Diante disso, observa-se que algumas das maiores dificuldades do trabalho em equipe são a relação interpessoal, a relação de poder e, até mesmo, as distintas concepções do que seja trabalhar em equipe, o que demonstra a importância da dimensão do trabalho enquanto interação social no campo da saúde (CARVALHO, 2012).

Trabalho em equipe significa construir consensos, saberes e práticas em cada profissional. Nele, são criados os vínculos entre os profissionais, sendo a maneira mais adequada de resolver os problemas. Significa, também, a utilização das interações entre os agentes envolvidos, o reconhecimento recíproco de saberes e da autonomia técnica (MARTINS ET AL., 2012).

No contexto da APS, especificamente nas unidades de saúde da família, cada 
profissional deve desempenhar a profissão em um processo de trabalho coletivo, de prática compartilhada, divisão de responsabilidades e de cuidado entre os membros da equipe, pautada nas contribuições específicas das diversas áreas de conhecimento (COSTA ET AL., 2012).

Nesse sentido, pensar os determinantes como: a autonomia, o vínculo entre a equipe multiprofissional na busca da resolubilidade, no contexto do cuidado, exige construção de novas práticas, reordenamento do modelo de atenção e acolhimento no fazer cotidiano da rede de serviços de saúde, das necessidades dos usuários (CAMPOS; DOMITI, 2007).

\section{Encaminhamentos e dificuldades na contrarreferência: (des)articulação dos níveis de complexidade do SUS.}

A partir da análise dos discursos dos participantes da pesquisa, foi possível observar que o fluxo singular no sistema de saúde tem início com a entrada na atenção primária, a partir de uma assistência qualificada. Da mesma forma, nas atenções secundária e terciária, faz-se necessário que a equipe sempre atrele ações que favoreçam todos os níveis de atenção, nos quais o profissional deve agir com autonomia durante todo o seu percurso.

[...] a gente tenta dar suporte a todos os níveis

[...] tenta atuar em todos os níveis de complexidade [...] na parte secundária, quando a doença está no início ainda, pra gente não deixar desenvolver, e na parte terciária, quando a complexidade já esta alta demais. (Terapeuta Ocupacional Nasf).

Portanto, percebe-se que a resolubilidade do cuidado está vinculada à atuação profissional em todos os níveis de complexidade. Desse modo, observa-se a diminuição da dicotomia entre os serviços de ações de saúde pública e aqueles responsáveis pelas ações de assistência médica, estabelecida ao longo da constituição da rede de serviços, que teve início a partir do uso de medicamentos, exames e equipamentos biomédicos (CAMPOS; DOMITTI, 2007).

A integralidade, nesse sentido, inicia-se pela organização dos processos de trabalho na atenção primária, onde se preconiza a assistência multiprofissional, operando por meio de dispositivos, como o acolhimento e o vínculo, em que a equipe se responsabiliza pelo cuidado ao usuário em seu contexto mais amplo (FRANCO; FRANCO, 2010).

No entanto, apesar da valorização da atuação em todos os níveis de complexidade do sistema, um aspecto identificado pelos profissionais, que dificulta a efetivação desse fluxo, é a realização de referência e contrarreferência nos atendimentos na atenção primária. Esta, quando realizada de forma efetiva e coerente, com acompanhamento do caso, é vista como algo relevante na articulação da rede. Contudo, vale ressaltar que, no cotidiano dos serviços, essa lógica de organização ainda é pouco utilizada, como se percebe no discurso:

[...] o paciente que precisa de uma atenção maior, de um atendimento secundário ou terciário, ele é referenciado [...] o paciente é encaminhado, depois atendido e depois ele retorna. A dificuldade maior seria essa contrarreferência. Muitas vezes, na maioria das vezes, os profissionais especialistas do atendimento secundário e terciário não fazem essa contrarreferência [...]. (Médica ESF).

[...] nós temos um grande problema na contrarreferência, porque, muitas vezes, o profissional não nos retorna com os resultados [...] essa contrarreferência nunca é feita pelo médico. (Enfermeira ESF).

Também há problemas para a efetivação da referência, pois sabe-se que cada serviço de atenção tem responsabilidades sobre o cuidado prestado, e deve haver um acompanhamento, garantindo o acesso aos outros níveis de assistência, caso não haja a especialidade desejada pelo usuário. No entanto, observa-se, a partir 
dos discursos dos profissionais, a fragilidade do acesso e a grande fila de espera para os atendimentos de especialidades, além dos encaminhamentos sem resolutividade.

[...] gente chega aqui é de partir o coração. Precisa de fisioterapia e a gente diz: vou passar você para o médico e o médico Ihe encaminha. E eles respondem: não, minha filha. Não adianta encaminhar, não, porque é em outro bairro. (Enfermeira PSF).

[...] entra governante, sai governante e a gente pensa: tomara que um dia apareça alguém pra resolver essa questão da fila de espera de encaminhamentos. (Enfermeira PSF).

Nesse contexto, em vez de processos de referência e contrarreferência, na prática, ocorrem encaminhamentos para serviços mais especializados, onde não se observa $o$ acompanhamento do paciente pala equipe da Unidade Básica de Saúde e nem a longitudinalidade da assistência prestada, dificultando a integralidade do cuidado.

O sistema de referência e contrarreferência é uma forma de organização dos serviços de saúde, que possibilita o acompanhamento profissional do usuário e favorece o seu acesso a todos os níveis assistenciais existentes no SUS. Para sua efetivação, é necessário que as unidades do sistema estejam organizadas hierarquicamente, isto é, classificadas pelo tipo de serviços, equipamentos e profissionais especialistas e a capacidade de resolutividade (ORTIGA, 2006).

A referência representa a necessidade do usuário de um maior grau de complexidade, de onde é encaminhado a um atendimento de especialização mais complexo, como hospitais e clínicas especializadas. Já a contrarreferência diz respeito ao menor grau de complexidade, quando a necessidade do usuário, com relação aos serviços de saúde, é mais simples, ou seja, ele é conduzido para um atendimento em nível primário, devendo ser a unidade de saúde mais próxima de seu domicílio (FRATINI; SAUPE, 2008).

Desse modo, o sistema de referência e contrarreferência é entendido como um mecanismo de encaminhamento mútuo de usuários entre os diferentes níveis de complexidade dos serviços. O Ministério da Saúde define esse sistema, inclusive, como um dos elementos-chave de reorganização das práticas de trabalho, e pode ser visto como o elemento para a integração das redes de saúde (SERRA; RODRIGUES, 2010).

Entretanto, Franco e Franco (2010) defendem o conceito de Unidades de Produção de Cuidado, que podem estar integradas e operando em um mesmo processo produtivo ou atuar de forma compartimentada, autonomizadas umas em relação às outras, baseados no Projeto Terapêutico como fio condutor do processo de cuidado. Essa forma de se relacionar em prol da constituição desse projeto fundamentado na demanda do usuário, a partir de olhares múltiplos, sustentados por diversos saberes, pode dar a liga entre os diversos produtos das Unidades, funcionando como uma cadeia produtiva dos Projetos Terapêuticos, levando aos resultados esperados no tocante à resolução do problema de saúde do usuário.

Com efeito, o Projeto Terapêutico pode constituir o fio condutor para os fluxos da linha do cuidado, os quais devem ser capazes de garantir o acesso seguro às tecnologias necessárias à assistência. Assim, o fluxo parte da rede básica, ou de qualquer outro lugar de entrada no sistema, para os diversos níveis assistenciais (FRANCO; FRANCO, 2010). Esse percurso dinâmico e autônomo dentro da rede pode funcionar de forma mais efetiva do que aquele operado na lógica de referência e contrarreferência, que parece obstruir a integralidade do cuidado.

Nesse sentido, as redes de saúde funcionam como uma comunicação de ações e serviços passíveis de transformação e reinvenção de papéis e territorialidades, fundamentais para a constituição e a operação 
de diferentes formas de cuidado. Essa articulação em rede rompe com o conceito de centralidade de cuidados, ou seja, oferece dinamismo e flexibilidade aos serviços, a fim de gerar melhores resultados da atenção.

Outro aspecto importante percebido é o de que os trabalhadores da atenção primária conhecem as redes de saúde, no entanto, ainda há dificuldades para o desenvolvimento de um trabalho articulado em todos os níveis de atenção, como se observa na fala dos profissionais da ESF:

[...] nós temos conhecimento de todas as redes [...] sabemos que precisa apenas melhorar ainda mais [...] Eu digo para o meu paciente: - Parabéns! Você está com o melhor plano de saúde que você já teve na sua vida. (Médica ESF).

[...] a gente só encaminha especialidades, porque aqui só tem o básico. [...] nunca encaminha e volta. Só quando lá está cheio e surge alguma urgência eles mandam. (Dentista ESF).

O profissional que tem o conhecimento da rede apresenta um posicionamento comprometido e implicado no cuidado dos sujeitos. Todo profissional de saúde, independentemente do papel que desempenhe como produtor de atos de saúde, é sempre um operador de cuidado, isto é, sempre atua clinicamente, e, como tal, deveria ser capacitado, pelo menos, para operar no campo específico das tecnologias leves, dos modos de produzir acolhimento, responsabilizações e vínculo. Pensá-los na direção de atos comprometidos com as necessidades do usuário (MERHY, 2002).

O conhecimento das redes de saúde envolve diversos aspectos inter-relacionados, tais como: regulação dos serviços; processos de gestão clínica; condições de acesso aos serviços; recursos humanos; sistemas de informação e comunicação. Pode-se considerá-los como condições necessárias também para o bom funcionamento do sistema de referência e contrarreferência (SERRA; RODRIGUES, 2010).
Desse modo, a utilização do arranjo em rede representa uma alternativa aos entraves do sistema de saúde. Por sua vez, a rede de cuidado visa a assegurar retaguarda especializada, tanto em nível assistencial como técnico-pedagógico. Pressupõe, assim, uma construção compartilhada entre a equipe de referência (profissionais da atenção primária) e os apoiadores, equipe de especialistas, com a missão de agregar conhecimentos, contribuindo para intervenções que aumentem sua capacidade de resolver problemas no nível da atenção primária (MARTINS ET AL., 2012).

Portanto, evidencia-se a importância de, não apenas conhecer a rede de saúde, mas, também, de buscar pautar suas ações de forma a garantir a articulação entre os serviços. Também se faz necessário procurar superar os obstáculos existentes no cotidiano dos serviços, que dificultam a resolubilidade.

Nesse cenário, fica evidente a importância da resolutividade para a satisfação dos usuários e para a qualidade dos serviços, pois é possível inferir que ter resolutividade, na maioria das vezes, está associado à satisfação. Assim, pode-se dizer que a promoção de resolutividade gera satisfação na clientela e na qualidade do atendimento em saúde.

\section{Considerações finais}

A resolutividade pode ser alcançada por meio de um atendimento acolhedor, mediante responsabilização das equipes, com atitudes criativas e flexíveis. Nesse sentido, o trabalho resolutivo em saúde baseia-se no cuidado corresponsável, em que prevaleça o protagonismo da equipe multiprofissional, no sentido de aprofundar os saberes e as práticas no campo da saúde. Essa ação pressupõe produção de vínculos interpessoais e contratuais, além de autonomia no processo de trabalho na atenção primária.

Outro aspecto que deve ser destacado é o modelo de redes de cuidado na articulação dos níveis assistenciais do SUS. A partir de 
uma atuação das equipes de base territorial, essa organização em rede promove o estabelecimento de vínculos e acolhimento. No entanto, na prática, observam-se dificuldades no processo de trabalho no primeiro nível de atendimento, especificamente, na incompletude das equipes, nos encaminhamentos e na contrarreferência.

$\mathrm{O}$ entendimento sobre resolutividade da assistência é fundamental para a reorganização da qualidade dos serviços de saúde, utilizando-se a análise de satisfação do usuário. Assim, profissionais de saúde e gestores podem realizar autoanálise de seus processos de trabalho e propor intervenções mais adequadas, em função das necessidades e expectativas sobre o serviço.

\section{Referências}

ANDRADE, L. M. et al. Análise da implantação dos Núcleos de Apoio à Saúde da Família no interior de Santa Catarina. Rev. Saúde e Transformação, Florianópolis, v. 3, n. 1, p. 18-31, 2012.

ASSIS, M. A. A.; JORGE, M. S. B. Métodos de análise em pesquisa qualitativa. In: SANTANA, J. S. S. S.; NASCIMENTO, M. A. A. Pesquisa: métodos e técnicas de conhecimento da realidade social. Feira de Santana: UEFS, 2010, p. 139-159.

CAMPOS, G. W. S.; DOMITTI, A. C. Apoio matricial e equipe de referência: uma metodologia para gestão do trabalho interdisciplinar em saúde. Cad. Saúde Pública, Rio de Janeiro, v. 23, n. 2, p. 399-407, 2007.

CARVALHO, G. Coordenação de unidade de atenção básica no SUS: trabalho, interação e conflitos. 2012. 300 f. Tese (Doutorado em Enfermagem). - Escola de Enfermagem da Universidade de São Paulo, São Paulo, 2012.

COSTA, M. R. et al. O trabalho em equipe desenvolvido pelo cirurgião dentista na Estratégia Saúde da Família: expectativas, desafios e precariedades. Revista Brasileira de Medicina de Família e Comunidade, Rio de Janeiro, v. 7, n. 24, p.147-163, 2012.

FERREIRA, R. C. et al. Trabalho em equipe multiprofissional: a perspectiva dos residentes médicos em saúde da família. Ciênc. Saúde Coletiva, Rio de
Janeiro, v.14, suppl.1, p. 1421-1428, 2009.

FRANCO, C. M.; FRANCO, T. B. Linhas do cuidado integral: uma proposta de organização da rede de saúde. 2010. Disponível em: <http://www.saude.rs.gov. br/dados/1312992014173Linha-cuidado-integralconceito-como-fazer.pdf $>$. Acesso em: 20 julho 2014.

FRATINI, J. R. G; SAUPE, G. Referência e contrarreferência: contribuição para a integralidade em saúde. Cienc. Cuid. Saude, Maringá, v. 7, n.1, p. 65-72, 2008.

GONCALVES, R. J. et al. Ser médico no PSF: formação acadêmica, perspectivas e trabalho cotidiano. Rev. Bras. Educ. Med, Rio de Janeiro, v. 33, n. 3, p. 382-392, 2009.

JULIANI, C. M. C. M.; CIAMPONE, M. H. T. Organização do sistema de referência e contrareferência no contexto do sistema único de saúde: a percepção de enfermeiro. Rev. Esc. Enferm. USP, São Paulo, v. 33, n. 4, p. 323-333, 1999.

LIMA, I. F. S. et al. Integralidade na percepção dos trabalhadores de uma unidade básica de saúde da família. Rev. Esc. Enferm. USP, São Paulo, v. 46, n. 4, p. 944-952, 2012

MARTINS, A. R. et al. Relações interpessoais, equipe de trabalho e seus reflexos na atenção básica. Rev. Bras. 
Educ. Med, Rio de Janeiro, v. 36, n. 1, p. 6-12, 2012.

MEDEIROS, C. R. G. et al. A rotatividade de enfermeiros e médicos: um impasse na implementação da Estratégia de Saúde da Família. Ciênc. Saúde Coletiva, Rio de Janeiro, v. 15, supl. 1, p. 1521-1531, 2010.

MENDES, E. V. As redes de atenção à saúde. Ciênc. Saúde Coletiva, Rio de Janeiro, v. 15, n. 5, p. 2297-2305, 2010.

MERHY, E. E.; ONOCKO, R. (Org.). Agir em saúde: um desafio para o público. São Paulo: Hucitec, 1997.

MERHY, E. E. A cartografia do trabalho vivo. 3. ed. São Paulo: Hucitec, 2002.

MINAYO, M. C. S. (Org.). Pesquisa social: teoria, método e criatividade. 14. ed. Petrópolis: Vozes, 1999.

O desafio do conhecimento: pesquisa qualitativa em saúde. 5. ed. Rio de Janeiro: Hucitec, 2008.

NUNES, A. A. et al. Resolubilidade da Estratégia Saúde da Família e Unidades Básicas de Saúde Tradicionais: Contribuições do PET-Saúde. Rev. Bras. Educ. Med, Rio de Janeiro, v. 36, n. 1, supl. 1, p. 27-32; 2012.

ORTIGA, A M. B. Estrutura e dinâmica das unidades de saúde. 2006. Mimeo.

PINTO, E. S. G. et al. Situação de trabalho dos profissionais da Estratégia Saúde da Família em CearáMirim. Rev. Esc. Enferm. USP, São Paulo, v. 44, n. 3, p. 657-664, 2010.

PIRES, R. O. M; CAMPOS, D. A. C. Equipe

Multiprofissional em Saúde da Família: do Documental ao Empírico no Interior da Amazônia. Rev. Bras. Educ. Med, Rio de Janeiro, v. 34, n. 3, p. 379-389, 2010.

POZ, M. R. D. A crise da força de trabalho em saúde. Cad. Saúde Pública, Rio de Janeiro, v. 29, n. 10, p. 1924-1926, 2013

QUINDERE, P. H. D. et al. Acessibilidade e resolubilidade da assistência em saúde mental: a experiência do apoio matricial. Ciênc. Saúde Coletiva, Rio de Janeiro, v. 18, n. 7, p. 2157-2166, 2013.

RODRIGUES, J. S. M. et al. O atendimento por instituição pública de saúde: percepção de famílias de doentes com câncer. Saúde em Debate, Rio de Janeiro, v. 37 , n. 97 , p. $270-280,2013$.

SCHRAIBER, L. B. et al. Planejamento, gestão e avaliação em saúde: identificando problemas. Ciênc. Saúde Coletiva, Rio de Janeiro, v. 4, n. 2, p. 221-42, 1999.

SERRA, C. G; RODRIGUES, P. H. A. Avaliação da referência e contrarreferência no Programa Saúde da Família na região metropolitana do Rio de Janeiro. Ciênc. Saúde Coletiva, Rio de Janeiro, v.15, supl. 3, p. 3579-86, 2010.

\section{SHIMIZU, H. E.; CARVALHO JUNIOR, D. A. C. O} processo de trabalho na Estratégia Saúde da Família e suas repercussões no processo saúde-doença. Ciênc. Saúde Coletiva, Rio de Janeiro, v. 17, n. 9, p. 2405-2414, 2012.

SILVA, S. F. Organização de Redes regionalizadas e Integradas de Atenção à Saúde: Desafios do Sistema Único de Saúde (Brasil). Ciênc Saúde Coletiva, Rio de Janeiro, v. 16, n. 6, p. 2753-2762, 2011.

SILVA, A. T. C. et al. Núcleos de Apoio à Saúde da Família: desafios e potencialidades na visão dos profissionais da Atenção Primária do Município de São Paulo, Brasil. Cad. Saúde Pública, Rio de Janeiro, v. 28, n. 11, p. 2076-2084, 2012

TURRINI, R. N. T. et al. Resolutividade dos serviços de saúde por inquérito domiciliar: percepção do usuário. Cad. Saúde Pública, Rio de Janeiro, v. 24, n. 3, p. 663-674, 2008.

Recebido para publicação em abril de 2013

Versão final em agosto de 2014

Conflito de interesses: inexistente

Suporte financeiro: Produção do cuidado na Estratégia Saúde da Família e sua interface com a saúde mental: os desafios em busca da resolubilidade (Projeto CNPq) - no 500827/2010-9 